\title{
MiR-574-5p mediates the cell cycle and apoptosis in thyroid cancer cells via Wnt/ $\beta$-catenin signaling by repressing the expression of Quaking proteins
}

\author{
ZHEJIA ZHANG $^{1}$, XINYING LI ${ }^{1}$, QIAN XIAO ${ }^{2}$ and ZHIMING WANG ${ }^{1}$ \\ Departments of ${ }^{1}$ General Surgery and ${ }^{2}$ Mental Hygiene Clinics, Xiangya Hospital, \\ Central South University, Changsha, Hunan 410008, P.R. China
}

Received November 23, 2016; Accepted November 29, 2017

DOI: $10.3892 / \mathrm{ol} .2018 .8067$

\begin{abstract}
Thyroid cancer is the most frequently occurring type of endocrine tumor, with a rapidly increasing incidence rate. MicroRNA (miR)-574-5p is a candidate oncogene in various types of cancer. The present study identified that miR-574-5p affected the cell cycle distribution and apoptosis of BCPAP and FTC133 thyroid cancer cells via $\beta$-catenin/Wnt signaling by targeting Quaking proteins (QKIs). An MTT assay demonstrated that the knockdown of miR-574-5p suppressed the proliferation of the thyroid cancer cells. Fluorescence-activated cell sorting analysis demonstrated that the inhibition of miR-574-5p induced the $G_{1} / S$ phase arrest and apoptosis of the cells. Reverse transcription-quantitative polymerase chain reaction and western blot analyses revealed that the knockdown of miR-574-5p significantly upregulated the mRNA and protein expression levels of QKIs. Furthermore, western blot analysis identified that the knockdown of miR-574-5p also repressed the $\mathrm{Wnt} / \beta$-catenin pathway via downregulating the expression of $\beta$-catenin, cyclin D1 and survivin, and upregulating the phosphorylation of $\beta$-catenin. The further depletion of QKIs in combination with the knockdown of miR-574-5p not only increased the expression of $\beta$-catenin, cyclin D1 and survivin, but also rescued the apoptosis of thyroid cancer cells induced by the miR-574-5p knockdown. In conclusion, these findings indicated that the aberrant upregulation of miR-574-5p may be oncogenic, through regulating the $\mathrm{Wnt} / \beta$-catenin pathway by targeting QKIs.
\end{abstract}

Correspondence to: Mr. Zhiming Wang, Department of General Surgery, Xiangya Hospital, Central South University, 87 Xiangya Road, Changsha, Hunan 410008, P.R. China

E-mail: wangzhiming008@yahoo.com.cn

Key words: microRNA-574-5p, thyroid cancer, Wnt/ $\beta$-catenin signaling, Quaking proteins, cell cycle, apoptosis

\section{Introduction}

Thyroid cancer is the most frequently occurring tumor in the endocrine system and accounts for $\sim 1 \%$ of all newly diagnosed cancer cases in the United States (1). The incidence of thyroid cancer, particularly differentiated-type thyroid cancer, is rapidly increasing (2-5). Thyroid cancer is primarily derived from two thyroid cell types: Follicular thyroid cells and parafollicular cells. The follicular thyroid cell-derived tumors account for the majority of thyroid cancer cases, including anaplastic thyroid cancer, poorly differentiated thyroid cancer, differentiated papillary thyroid cancer (PTC) and follicular thyroid cancer (FTC). Medullary thyroid cancer (MTC), derived from parafollicular $\mathrm{C}$ cells, accounts for a relatively small proportion of thyroid cancers (6). Genetic and epigenetic alterations, including mutations, copy number variation and abnormal methylation, are the primary causative factors of the pathogenesis of thyroid cancer $(1,6)$. Well-differentiated PTC and FTC can be effectively treated by surgery followed by radioiodine therapy. Tumors without differentiation are candidates for molecularly targeted therapy, as they are associated with a less favorable prognosis (7).

Quaking (QKI) is an RNA-binding protein that belongs to the signal transduction and activation of RNA (STAR) protein family (8). QKI encodes a diverse set of transcript variants through alternative splicing, of which the most well-documented isoforms are QKI 5, 6 and 7 (9). QKI has been associated with regulating various cellular processes, including apoptosis, the cell cycle, differentiation and cell fate determination (10-12). The decreased expression of QKI has been implicated in the pathogenesis of many types of human disease, including a number of types of cancer $(8,13)$. Yang et al (14) reported that the levels of QKI5/6 were significantly reduced or absent in colorectal cancer (CRC) cells. It has also been observed that the downregulation of QKI in colon cancers may be partially due to the hypermethylation of the QKI promoter region. The induced expression of QKI decreases the cell proliferation ability and increases the differentiation ability of colon cancer cells (14).

MicroRNAs (miRNA) are small noncoding RNAs that function in RNA splicing and the post-transcriptional regulation of gene expression via targeting 
complementary sequences within mRNA to induce mRNA degradation (15-17). The dysregulation of miRNAs and their associated target genes may participate in tumorigenesis $(18,19)$. miR-574-5p is a potential oncogenic miRNA that has been demonstrated to be significantly upregulated in thyroid cancer cells $(18,19)$.

Wnt/ $\beta$-catenin signaling is constitutively activated in cancer cells. Phosphorylated $\beta$-catenin may be degraded by proteasomes. However, non-phosphorylated $\beta$-catenin is transferred into the nucleus and protected from degradation, thereby activating the expression of genes associated with cell proliferation (20). Thus, the phosphorylation level of $\beta$-catenin indicates the activity of the $\mathrm{Wnt} / \beta$-catenin pathway and promotes cancer cell proliferation.

Previous studies have demonstrated that miR-574-5p regulates CRC tumorigenesis and progression by targeting QKI and subsequently activating the Wnt/ $\beta$-catenin pathway (8). However, it remains unclear the mechanism by which miR-574-5p regulates QKI and functions as an oncogene in thyroid cancer.

In the present study, miR-574-5p-specific small interfering RNA (siRNA) was utilized to decrease the miR-574-5p level, and the influence of miR-574-5p on cell proliferation, the cell cycle and apoptosis was analyzed in BCPAP and FTC133 thyroid cancer cells. The expression of QKI and crucial factors of the Wnt/ $\beta$-catenin pathway following miR-574-5p knockdown in thyroid cell lines were also detected. The results of the present study suggested that miR-574-5p induced cell proliferation and cell cycle progression, and repressed the apoptosis of thyroid cancer cells via $\mathrm{Wnt} / \beta$-catenin signaling by targeting QKIs.

\section{Materials and methods}

Cell culture and siRNA transfection. The human thyroid cells BCPAP and FTC133 (American Type Culture Collection, Manassas, VA, USA) were cultured in Dulbecco's modified Eagle's medium (DMEM; Gibco; Thermo Fisher Scientific, Inc., Waltham, MA, USA) with $10 \%$ fetal bovine serum (FBS; Gibco, Thermo Fisher Scientific, Inc.) at $37^{\circ} \mathrm{C}$ with $5 \% \mathrm{CO}_{2}$ in an incubator. A total of $2 \times 10^{5}$ BCPAP or FTC133 cells were plated into 6-well dishes. At $24 \mathrm{~h}$ after seeding, siRNA targeting miRNA-574 or QKI, or negative control siRNAs were transfected into cells using Lipofectamine ${ }^{\circledR} 2000$ (Invitrogen, Thermo Fisher Scientific, Inc.) according to the manufacturer's protocol. Cells transfected with the miR-574-5p siRNA or negative control siRNA were used for proliferation, cell cycle, apoptosis, reverse transcription-quantitative polymerase chain reaction (RT-qPCR) and western blotting assays. Cells transfected with miR-574-5p siRNA and QKI siRNA were used for apoptosis and western blotting assays. The miR-574-5p-specific siRNA was purchased from Ambion (Thermo Fisher Scientific, Inc.; sequence 5'-UGAGUGUGU GUGUGUGAGUGUGU-3'), a scrambled sequence, as previously described (8), was used as negative control. The siRNA for pan-QKI knockdown was synthesized by Shanghai Sangon Pharmaceutical Co., Ltd. (Shanghai, China) with the sequence 5'-CCUUGAGUAUCCUAUUGAACCUAGU-3', whereas the negative control sequence was 5'-UUCUCCGAACGUGUC ACGUTT-3'.
Cell proliferation assay. Cell proliferation was detected by an MTT assay (Sigma-Aldrich, Merck KGaA, Darmstadt, Germany) according to the manufacture's protocol, with some modification, as previously described (21). At 24, 48, and $72 \mathrm{~h}$ after transfection, BCPAP and FTC133 cells were treated with MTT (5 mg/ml in $1 \mathrm{X}$ PBS), in an amount equal to $10 \%$ of the culture volume, for $4 \mathrm{~h}$. Absorbance at $570 \mathrm{~nm}$ was then detected. Three independent experimental repeats were performed.

Cell cycle assay. BCPAP and FTC133 cells transfected with miR-574-5p or negative control siRNA were collected, washed twice with $1 \mathrm{X}$ PBS, and fixed in $70 \%$ ethanol at $-20^{\circ} \mathrm{C}$. After $24 \mathrm{~h}$ of fixation, cells were incubated with RNase A (Takara Bio, Inc., Otsu, Japan) at $100 \mu \mathrm{g} / \mathrm{ml}$ in $1 \mathrm{X}$ PBS for $30 \mathrm{~min}$ at $37^{\circ} \mathrm{C}$. Cells were then stained with propidium iodide (PI; BD Biosciences, San Jose, CA, USA) at $50 \mu \mathrm{g} / \mathrm{ml}$ for $30 \mathrm{~min}$ at room temperature. Subsequently, cells were analyzed for DNA content using a BD FACSCalibur ${ }^{\mathrm{TM}}$ flow cytometer (BD Biosciences). Three independent experimental repeats were performed.

Apoptosis analysis. BCPAP and FTC133 cells transfected with miR-574-5p, QKI or negative control siRNA were collected for apoptosis analysis. An Annexin V-FITC PI staining assay kit (BD Biosciences) was used for the detection of apoptotic cells according to the manufacturer's protocol. Three independent experimental repeats were performed.

RNA extraction and RT- $q P C R$ analyses for miRNA and $m R N A s$. The total RNA of BCPAP and FTC133 cells transfected with miR-574-5p siRNA or negative controls was extracted using an RNeasy Mini kit (Qiagen, Valencia, CA, USA) according to the manufacturer's protocol. For mRNA quantification, RT was performed with the PrimeScript Reverse Transcriptase (Takara Bio) using a random primer mix (Invitrogen). qPCR assays were performed with the SYBR Premix Ex Taq ${ }^{\mathrm{TM}}$ (Takara Bio) on an ABI Prism 7500 Real-time PCR system (Thermo Fisher Scientific). DNA was denatured at $94^{\circ} \mathrm{C}$ for $10 \mathrm{~min}$, followed by initial denaturation with 30 cycles at $94^{\circ} \mathrm{C}$ for $1 \mathrm{~min}, 60^{\circ} \mathrm{C}$ for $1 \mathrm{~min}$ and $72^{\circ} \mathrm{C}$ for $2 \mathrm{~min}$, and finally ended up with an extension step at $72^{\circ} \mathrm{C}$ for $5 \mathrm{~min}$. The relative expression of genes was calculated by $2^{-\Delta \Delta \mathrm{Ct}}$ method (22). Each assay was performed three times.

For miR-574-5p quantification, RT was performed with the PrimeScript Reverse Transcriptase (Takara Bio) using the miR-574-5p-specific RT primer (Shanghai Sangon Pharmaceutical, Shanghai, China). qPCR assays were performed using the previously described universal primer (Shanghai Sangon Pharmaceutical) and miR-574-5p-specific reverse locked nucleic acid primers (LNA; Shanghai Sangon Pharmaceutical) $(8,23)$.

The primers were as follows: Pan-QKI forward: 5'-CAT CAGCTGCATCTTCTTCAG-3', pan-QKI reverse: 5'-CAC TGTGGAAGATGCTCAGAA-3'; QKI5 forward: 5'-GCC CTACCATAATGCCTTTGA-3', QKI5 reverse: 5'-AACTTT AGTAGCCACCGCAACC-3'; QKI6 forward: 5'-GCCCGA AGCTGGTTTAATCTATA-3', QKI6 reverse: 5'-TCGTTG GGAAAGCCATACCTAAT-3'; QKI7 forward: 5'-GCTGGT TTAATCTATACACCCTATGA-3', QKI7 reverse: 5'-GAC 


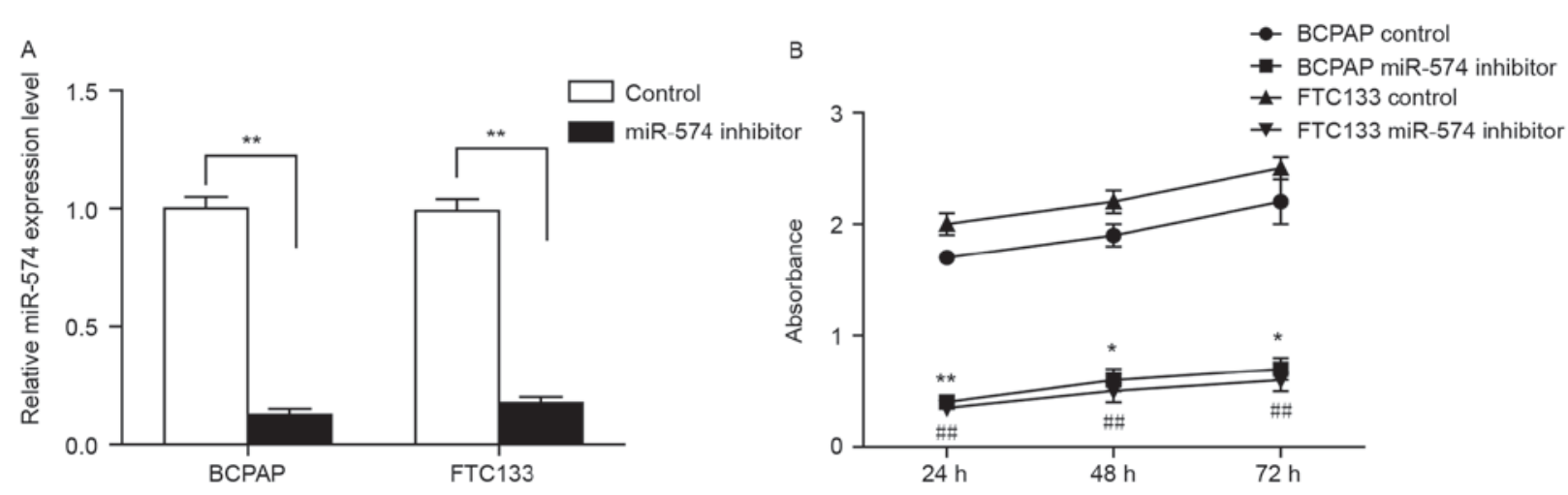

Figure 1. Knockdown of miR-574-5p decreased the proliferation of thyroid carcinoma cells. (A) Results of the reverse transcription-quantitative polymerase chain reaction assay, indicating a significant reduction of the miR-574-5p level in BCPAP and FTC133 cells transfected with the miR-574-5p siRNA. ** P $<0.01$. (B) MTT assay performed at the indicated time points from knockdown (24,48 and $72 \mathrm{~h}$ ) in BCPAP and FTC133 cells. The absorbance at $570 \mathrm{~nm}$ was detected after a 4-h incubation with MTT. Error bars show the standard deviation of triplicate experiments. ${ }^{~} \mathrm{P}<0.05$ and ${ }^{* *} \mathrm{P}<0.01$ vs. BCPAP control group. ${ }^{\# \prime} \mathrm{P}<0.01$ vs. FTC133 control group. miR, microRNA.

TGGCATTTCAATCCACTCTA-3'; miR-574-5p-specific RT primer: 5'-GTCGTATCCAGTGCGTGTCGTGGAGTCGGC AATTGCACTGGATACGACTACACAC-3'; universal primer, GGGGTGAGTGTGTGTGTG-3'; miR-574-5p-specific reverse LNA, TGCGTGTCGTGGAGTC-3'.

Western blotting. Total protein extracts from BCPAP and FTC133 cells with or without miR-574-5p knockdown were used for western blotting analysis, as previously described (8). The primary antibodies used for western blot analysis were as follows: Anti-pan-QKI (cat. no. sc103851; Santa Cruz Biotechnology, Inc., Dallas, TX, USA); anti-QKI5 (cat. no. AB9904); anti-QKI6 (cat. no. AB9906); anti-QKI7 (cat. no. AB9908; all EMD Millipore, Billerica, MA, USA); anti- $\alpha$-Tubulin (cat. no. 2125); anti- $\beta$-catenin (cat. no. 8480 ); anti-phospho- $\beta$-catenin (Ser33/37/41; cat. no. 9561); anti-cyclin D1 (cat. no. 2978); and anti-survivin (cat. no. 2808; all Cell Signaling Technology, Danvers, MA, USA). Each western blotting assay was performed three times.

Statistical analysis. Statistical analysis was performed using GraphPad Prism 5.0 (GraphPad Software, Inc., La Jolla, CA, USA). Statistical evaluation was performed using Student's t test (two-tailed) between two groups, or one-way analysis of variance followed by Tukey's post hoc test for multiple comparisons. $\mathrm{P}<0.05$ was considered to indicate a statistically significant difference.

\section{Results}

Inhibition of miR-574-5p suppresses the proliferation of BCPAP and FTC133 cells. To investigate the role of miR-574-5p in thyroid cancer, siRNA was used to knockdown miR-574-5p in the thyroid carcinoma-derived cell lines BCPAP and FTC133. The reduction of miR-574-5p expression in miR-574-5p siRNA-transfected cells was confirmed by RT-qPCR (Fig. 1A). Subsequently, an MTT assay was conducted to investigate any changes in cell growth following miR-574-5p knockdown. As demonstrated in Fig. 1B, the viability of BCPAP and FTC133 cells transfected with miR-574-5p siRNA was significantly reduced when compared with the negative control, indicating that miR-574-5p may promote thyroid cancer cell growth.

Inhibition of miR-574-5p leads to the $G_{I} / S$-phase arrest of BCPAP and FTC133 cells. The effect of miR-574-5p knockdown on the cell cycle distribution was investigated. As demonstrated by Fig. 2, following miR-574-5p knockdown, the proportion of $\mathrm{G}_{0} / \mathrm{G}_{1}$-phase cells was significantly increased, from $\sim 25$ to $50 \%$ (BCPAP) and from $\sim 26$ to $51 \%$ (FTC133), whereas the proportion of $\mathrm{S}$-and $\mathrm{G}_{2} / \mathrm{M}$-phase cells was significantly decreased. This result implied that the knockdown of miR-574-5p led to cell cycle arrest at the $\mathrm{G}_{1} / \mathrm{S}$ phase, suggesting that miR-574-5p promoted the cell cycle progression of thyroid cancer cells. Furthermore, the proportion of sub- $G_{0} / G_{1}$ phase cells was significantly increased following miR-574-5p knockdown, indicating the induction of apoptosis.

Inhibition of miR-574-5p induces the apoptosis of BCPAP and FTC133 cells. The cell cycle analysis indicated that the inhibition of miR-574-5p may affect the apoptotic status of thyroid carcinoma cells. Thus, the proportion of early and late apoptotic cells following miR-574-5p knockdown were determined through Annexin V-FITC/PI staining. Fig. 3 demonstrated that, following miR-574-5p knockdown, the proportion of early apoptotic cells (Annexin $\mathrm{V}^{+} / \mathrm{PI}^{-}$) was increased from 0.91 to $31.39 \%$ for BCPAP cells, and from 0.48 to $21.36 \%$ for FTC133 cells. Furthermore, the proportion of late apoptotic and necrotic (Annexin $\mathrm{V}^{+} / \mathrm{PI}^{+}$) cells following miR-574-5p knockdown was increased from 2.11 to $5.24 \%$ for BCPAP cells, and from 1.20 to $6.15 \%$ for FTC133 cells.

Inhibition of miR-574-5p upregulates the level of pan-QKI and QKI5/6/7 in BCPAP and FTC133 cells. The mechanism of miR-574-5p in the development of thyroid carcinoma has yet to be characterized. A previous study demonstrated that the inhibition of miR-574-5p in CRC suppressed tumor growth by the activation of QKI proteins (8). Therefore, the present study further investigated whether the expression levels of QKIs were influenced by miR-574-5p in thyroid carcinoma. 
A

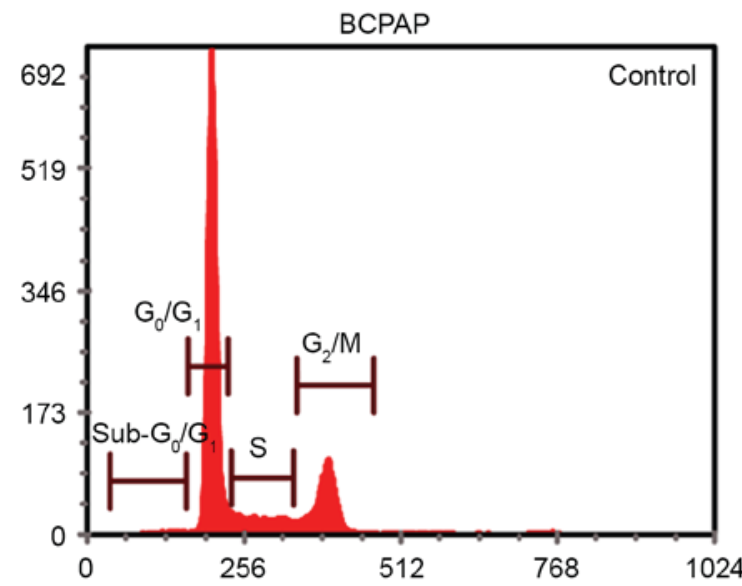

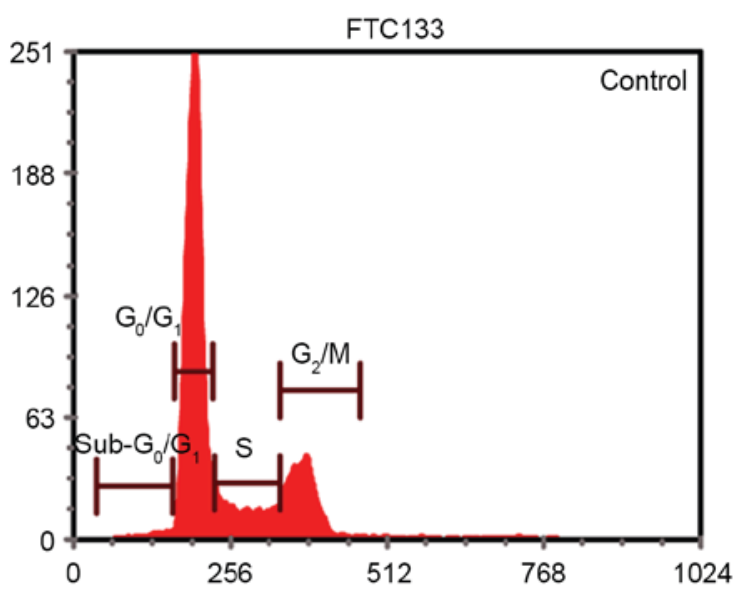
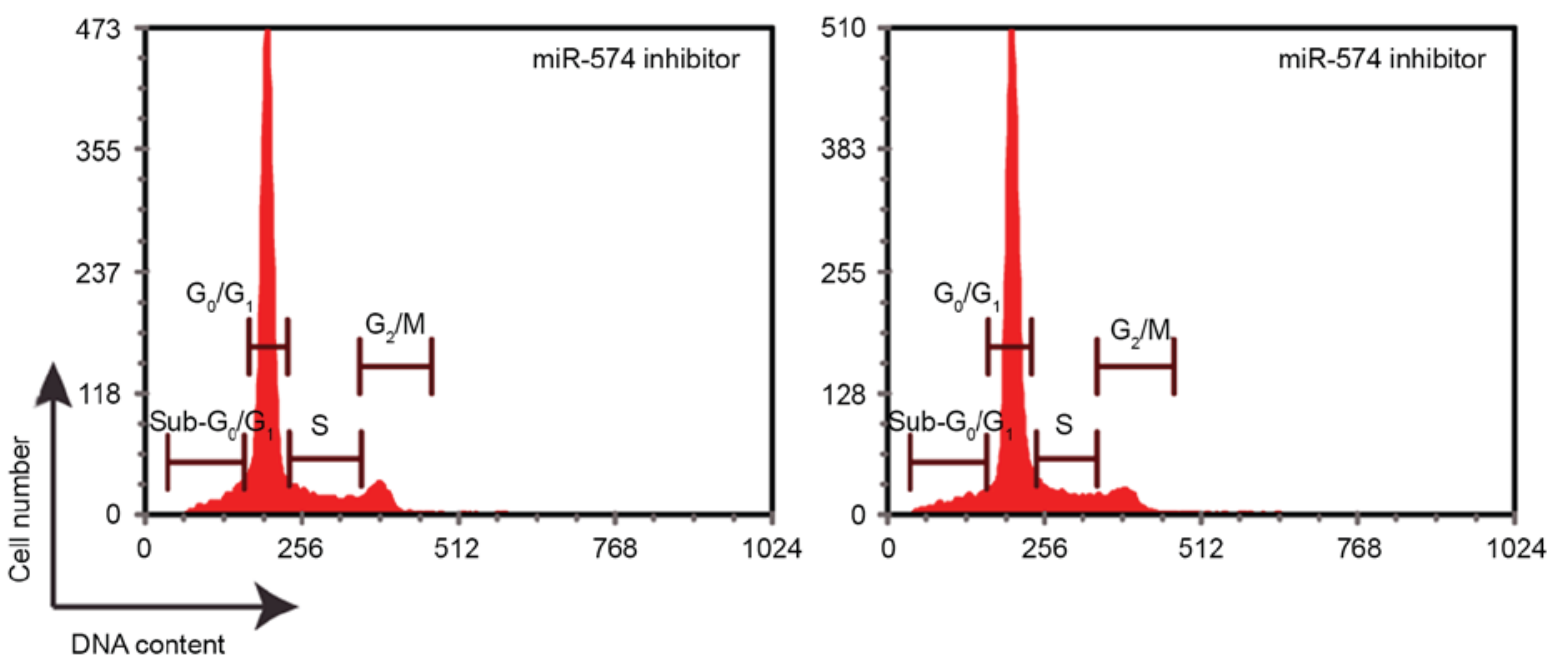

B

\&88 BCPAP control

B.

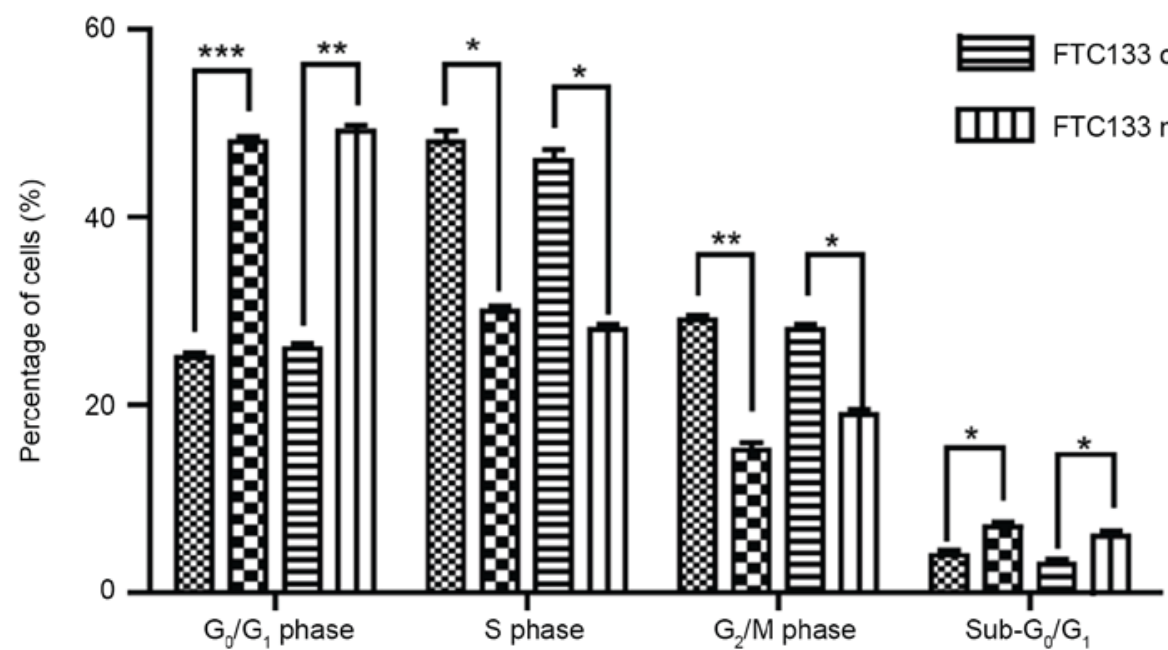

Figure 2. Knockdown of miR-574-5p led to cell cycle arrest at $\mathrm{G}_{1} / \mathrm{S}$ phase. (A) Histograms of the cell cycle distribution of BCPAP and FTC133 cells following miR-574-5p knockdown. (B) Proportion of cells in of each cell cycle phase following miR-574-5p knockdown. Data are presented as the mean \pm standard deviation of triplicate experiments. ${ }^{*} \mathrm{P}<0.05,{ }^{* *} \mathrm{P}<0.01$, and ${ }^{* * *} \mathrm{P}<0.001$ vs. respective control groups. miR, microRNA.

Initially, an RT-qPCR assay was performed to detect the level of pan-QKI and QKI5/6/7 mRNAs prior to and following the inhibition of miR-574-5p. As demonstrated in
Fig. 4A, the mRNA levels of pan-QKI and QKI5/6/7 were significantly higher in the miR-574-5p siRNA-transfected BCPAP and FTC133 cells compared with the negative control. 
A
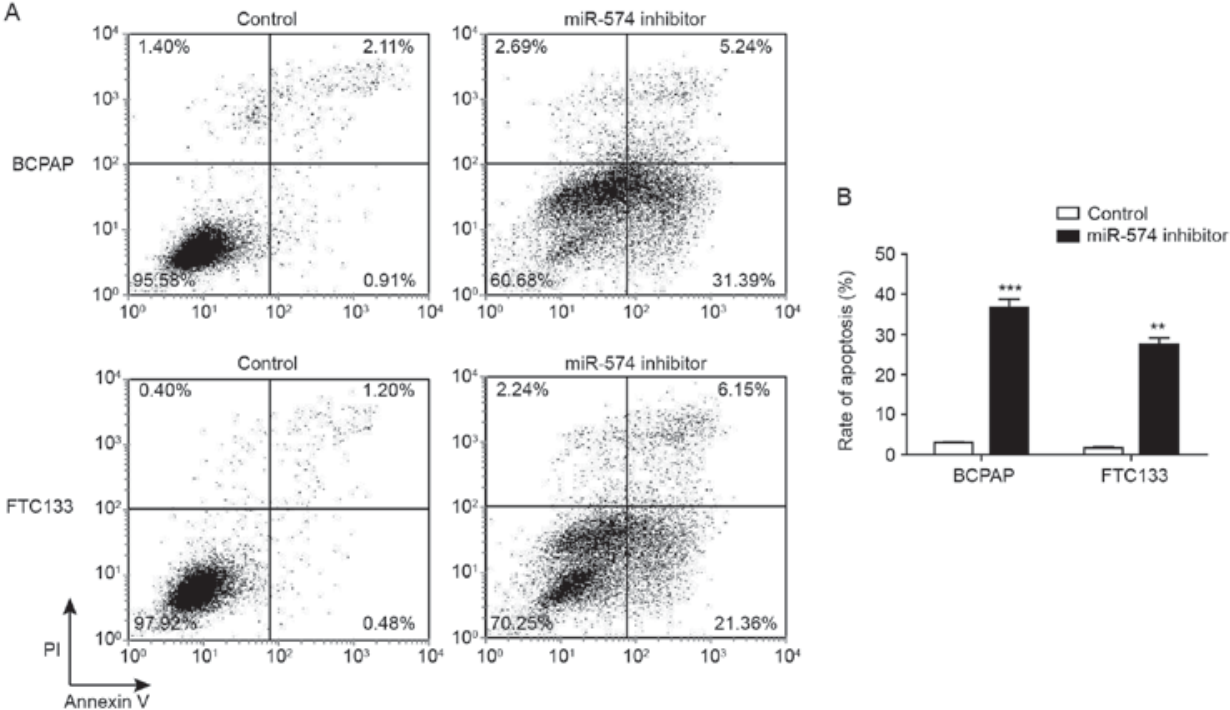

Figure 3. Knockdown of miR-574-5p induced apoptosis in BCPAP and FTC133 cells. Apoptosis was detected by flow cytometry following Annexin V/propidium iodide staining in BCPAP and FTC133 cells transfected with miR-574-5p siRNA and negative controls. (A) Representative dot plots from flow cytometric analysis. (B) Proportion of of apoptotic BCPAP and FTC133 cells with and without miR-574-5p knockdown. Data are presented as the mean \pm standard deviation of triplicate experiments. ${ }^{* *} \mathrm{P}<0.01$ and ${ }^{* * *} \mathrm{P}<0.001$ vs. respective control group. miR, microRNA.

A

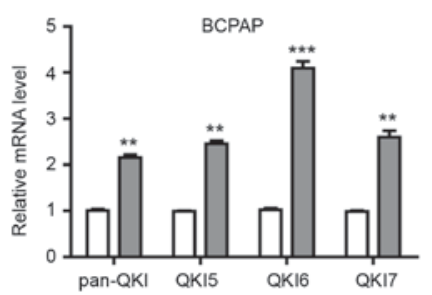

B

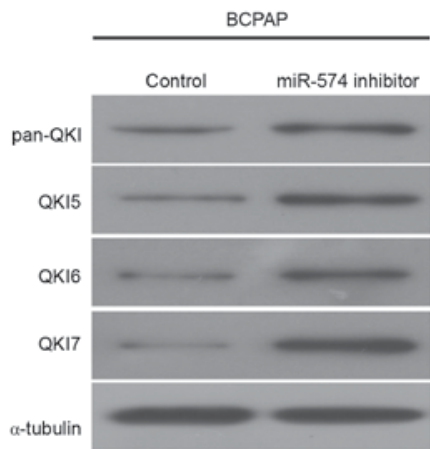

C

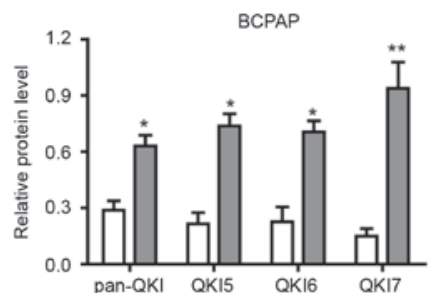

$\square$ control

$\square$ miR-574 inhibitor
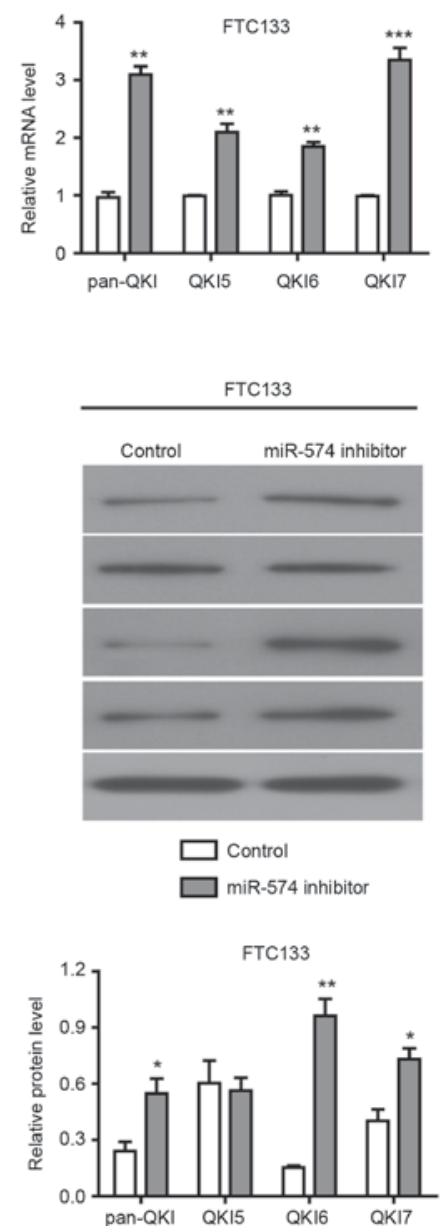

Figure 4. Knockdown of miR-574-5p significantly increased the expression level of QKIs in thyroid carcinoma cells. (A) mRNA level of pan-QKI and QKI5/6/7 detected in BCPAP and FTC133 cells transfected with miR-574-5p siRNA or a negative control. Data are presented the mean \pm standard deviation of triplicate experiments. ${ }^{* *} \mathrm{P}<0.01$ and ${ }^{* * *} \mathrm{P}<0.001$ vs. respective control groups. (B) Protein level of pan-QKI and QKI5/6/7 detected in BCPAP and FTC133 cells transfected with miR-574-5p siRNA or negative controls. $\alpha$-tubulin was used as an internal control. Images are representative of three western blot analysis repeats. (C) Quantification of western blotting assay. ${ }^{*} \mathrm{P}<0.05$ and ${ }^{* *} \mathrm{P}<0.01$ vs. respective control groups. miR, microRNA; QKI, quaking protein. 
A

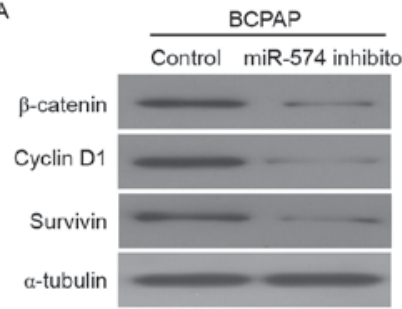

B
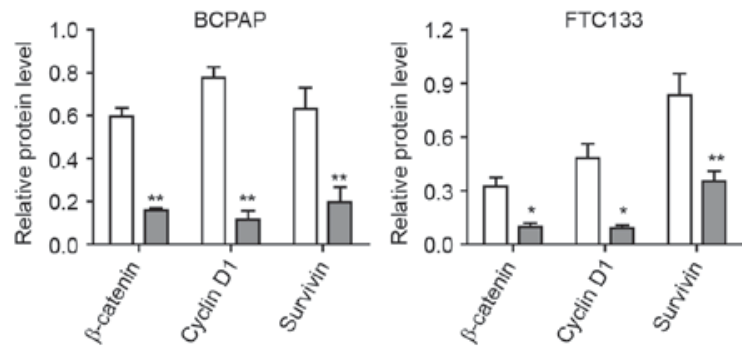

Figure 5. Knockdown of miR-574-5p repressed the Wnt/3-catenin pathway. (A) Western blotting results to demonstrate the change in protein expression levels following miR-574-5p knockdown. $\alpha$-tubulin was used as an internal control. Images are representative of three western blot analysis repeats. (B) Quantification of western blot assays. ${ }^{*} \mathrm{P}<0.05,{ }^{* *} \mathrm{P}<0.01$ vs. respective control group. miR, microRNA.

Subsequently, western blotting assays were conducted to assess the protein level of pan-QKI and QKI5/6/7. As demonstrated in Fig. 4B and C, the protein levels of pan-QKI and QKI5/6/7 were also increased following the miR-574-5p knockdown in BCPAP and FTC133 cells, with the exception of QKI5 protein in FTC133 cells. Collectively, these results indicated that miR-574-5p repressed the expression of QKIs at the mRNA and protein levels, suggesting that the influence of miR-574-5p on cell proliferation, cell cycle distribution and apoptosis may be mediated by the repression of QKIs.

Inhibition of miR-574-5p represses the $W n t / \beta$-catenin pathway. $\beta$-catenin is a core factor of the $\mathrm{Wnt} / \beta$-catenin signaling pathway and a downstream target of QKI proteins (24). As the expression of QKIs may be regulated by miR-574-5p, it was investigated in the present study how miR-574-5p affected the Wnt/ $\beta$-catenin signaling pathway through the western blot analysis of thyroid carcinoma cells. The knockdown of miR-574-5p led to the significant downregulation of $\beta$-catenin protein in BCPAP and FTC133 cells (Fig. 5), indicating that miR-574-5p may regulate $\mathrm{Wnt} / \beta$-catenin pathway via affecting the QKI proteins. Furthermore, the expression levels of two Wnt/ $\beta$-catenin target genes, cyclin D1 and survivin, were detected following miR-574-5p knockdown. As demonstrated in Fig. 5, the levels of cyclin D1, which regulated the cell cycle, and survivin, which regulated apoptosis, were significantly lower in miR-574-5p siRNA-treated BCPAP and FTC133 cells as compared with control siRNA-treated cells. Collectively, these results indicated that the depletion of miR-574-5p may lead to the repression of the Wnt/ $\beta$-catenin pathway.

miR-574-5p may regulate the Wnt/ $\beta$-catenin pathway by repressing $Q K I s$. The results of Fig. 4 demonstrate that QKIs, which have been reported to repress the Wnt/ $\beta$-catenin pathway, were repressed by miR-574-5p. In order to identify whether the repression of the Wnt/ $\beta$-catenin pathway subsequent to miR-574-5p knockdown was mediated by QKIs, the further knockdown of QKI expression was induced through siRNAs against all QKI isoforms. As illustrated in Fig. 6A and B, the expression levels of $\beta$-catenin, cyclin D1 and survivin were significantly increased following QKI knockdown in combination with miR-574-5p knockdown. Furthermore, the level of phosphorylated $\beta$-catenin was significantly upregulated following miR-574-5p knockdown in BCPAP and FTC133 cells. However, the level of phosphorylated $\beta$-catenin was decreased following QKI knockdown in miR-574-5p-knockdown BCPAP and FTC133 cells.

The biological effects of further QKI knockdown in miR-574-5p-knockdown BCPAP and FTC133 cells were also examined. Cells with the co-knockdown of QKIs and miR-574-5p exhibited a significant decrease in the rate of apoptosis when compared with the cells with only miR-574-5p knockdown (from 39.43 to $12.40 \%$ in BCPAP cells, and from 17.72 to $10.42 \%$ in FTC133 cells; Fig. 6C and D). Collectively, these results indicated that miR-574-5p may positively activate the Wnt/ $\beta$-catenin signaling pathway through repressing QKIs, and thus regulate the cell cycle and apoptosis.

\section{Discussion}

Thyroid cancer accounts for $\sim 1 \%$ of all newly diagnosed cancers in the United States, and is the most frequently occurring tumor in the endocrine system (1). miR-574-5p, a potential oncogenic miRNA, is upregulated in thyroid cancer (18). In the present study, it was demonstrated that miR-574-5p induced cell proliferation and cell cycle progression, and repressed the apoptosis of thyroid cancer cells via Wnt/ $\beta$-catenin signaling by downregulating QKIs. The inhibition of miR574-5p repressed proliferation and induced the apoptosis of thyroid cancer cells. These data may enhance the understanding of the association between the miR-574-5p and Wnt/ $\beta$-catenin pathway. Additionally, miR-574-5p may provide a novel potential therapeutic target for the treatment of thyroid cancer.

miRNAs may participate in tumorigenesis by acting as oncogenes or tumor suppressors $(16,25)$. miR-574-5p has been identified as upregulated in thyroid cancer cells (18). The present study demonstrated that the inhibition of miR-574-5p reduced the oncogenic behavior of thyroid cancer cells. Knockdown of miR-574-5p repressed the proliferation of thyroid cancer cells, led to cell cycle arrest at the $\mathrm{G}_{1} / \mathrm{S}$ phase, and increased the number of cells undergoing apoptosis. An increasing amount of evidence has indicated the oncogenic role of miR-574-5p. For example, miR-574-5p has been identified as aberrantly upregulated in CRC, and the inhibition of miR-574-5p suppressed the growth of tumors in murine models (8). Furthermore, miR-574-5p was identified as one of the most commonly upregulated miRNAs in lung cancer, and the repression of miR-574-5p significantly abrogated the tumor progression induced by Toll-like receptor 9 signaling (26). The present study provided evidence to support the oncogenic potential of miR-574-5p and the association between miR-574-5p and thyroid cancer.

QKI is associated with the determination of cell fate, including apoptosis, cell cycle progression and 


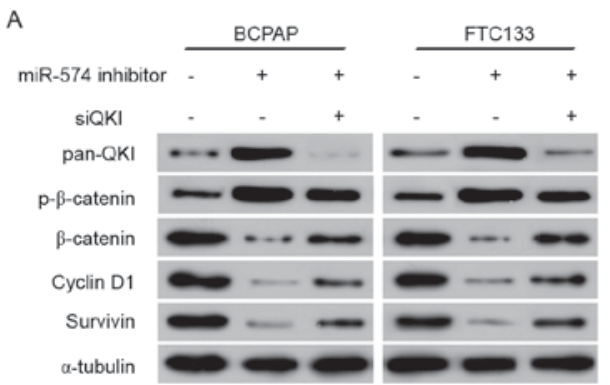

B
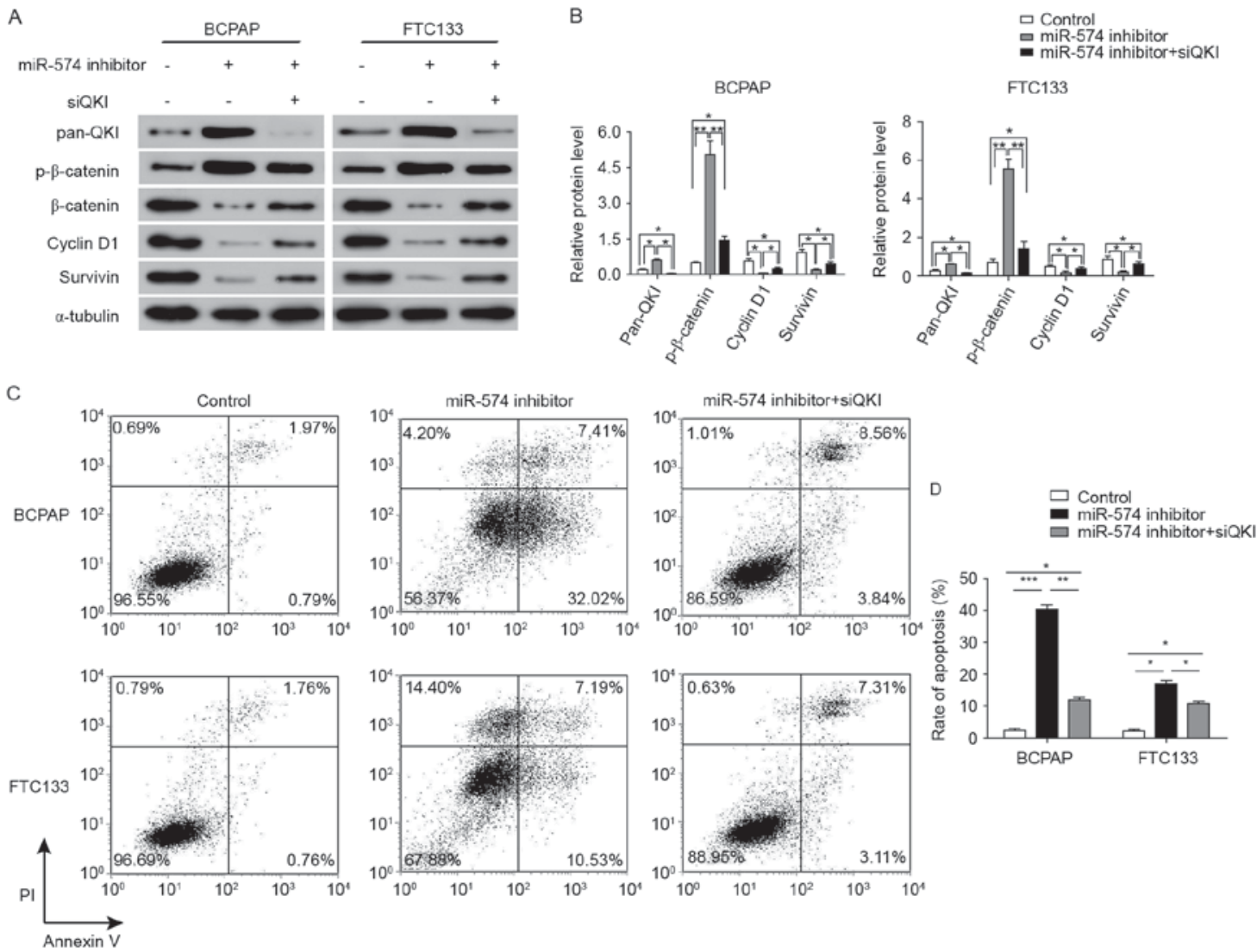

Figure 6. Knockdown of miR-574-5p repressed the Wnt/ $\beta$-catenin pathway through activating QKIs. (A) Western blotting showing the change of protein expression levels following miR-574-5p and QKI knockdown. $\alpha$-tubulin was used as an internal control. Images are representative of three western blot analysis repeats. (B) Quantification of western blotting. (C) Repression of QKIs rescued the cell apoptosis induced by miR-574-5p knockdown. (D) Quantification of the apoptosis rate in BCPAP and FTC133 cells. ${ }^{*} \mathrm{P}<0.05,{ }^{* *} \mathrm{P}<0.01,{ }^{* * *} \mathrm{P}<0.001$ vs. respective control groups. miR, microRNA; QKI, quaking protein.

differentiation $(13,27,28)$. The suppression of QKI, as may be induced by DNA methylation in the QKI promoter region (29), has been implicated in numerous cancer types $(8,30,31)$. In addition, miR-574-5p has been indicated to affect the regulation of QKI. For example, Yang et al (14) demonstrated that miR-574-5p negatively regulated QKI6/7 in CRC. In the present study, qPCR and western blotting assays showed that pan-QKI and QKI5/6/7 were significantly repressed by miR-574-5p in thyroid cancer. Furthermore, since QKI is a negative regulator of the $\mathrm{Wnt} / \beta$-catenin pathway, the influence of miR-574-5p on the Wnt/ $\beta$-catenin pathway was also investigated. The data indicated that miR-574 activated the expression of $\beta$-catenin through repressing QKIs. As a result, the knockdown of miR-574-5p led to the downregulation of total $\beta$-catenin and the upregulation of phosphorylated $\beta$-catenin. The expression of cyclin D1 and survivin, two important targets of the $\mathrm{Wnt} / \beta$-catenin pathway $(32,33)$, was also repressed by the knockdown of miR-574-5p. As a consequence, the repression of miR-574-5p led to the cell cycle arrest and apoptosis of the thyroid cancer cells. In summary, the present findings support the hypothesis that the oncogenic function of miR-574-5p is achieved through an effect on QKIs.

The primary contribution of the present study was to demonstrate the association between miR-574-5p and the oncogenic behavior of thyroid cancer cells. It was identified that the aberrantly high expression of miR-574-5p in thyroid cancer cells promoted cell proliferation and the cell cycle progression of thyroid cancer cells. Furthermore, the function of miR-574-5p in the cell cycle and apoptosis of thyroid cancer cells may be mediated via Wnt/ $\beta$-catenin signaling by the repression of QKIs. Thus, from a clinical perspective, these findings suggest that miR-574-5p may be a candidate therapeutic target for the treatment and prevention of thyroid cancer.

\section{Acknowledgements}

Not applicable.

\section{Funding}

This study was supported by National Natural Science Foundation of China (Grant number, 81372860).

\section{Availability of data and materials}

The datasets used and/or analyzed during the current study are available from the corresponding author on reasonable request.

\section{Authors' contributions}

Study concepts and design, ZJZ and ZMW; Literature research, XYL; Experimental studies and data analysis, ZJZ and QX; Manuscript editing and review, ZJZ, XYL, QX and ZMW. 


\section{Ethics approval and consent to participate}

Not applicable.

\section{Consent for publication}

Not applicable.

\section{Competing interests}

The authors declare that they have no competing interests.

\section{References}

1. Nikiforov YE: Thyroid carcinoma: Molecular pathways and therapeutic targets. Mod Pathol 21 (Suppl 2): S37-S43, 2008.

2. Jemal A, Siegel R, Ward E, Murray T, Xu J and Thun MJ: Cancer statistics, 2007. CA Cancer J Clin 57: 43-66, 2007.

3. Pacini F, Schlumberger M, Dralle H, Elisei R, Smit JW and Wiersinga W; European Thyroid Cancer Taskforce: European consensus for the management of patients with differentiated thyroid carcinoma of the follicular epithelium. Eur J Endocrinol 154: 787-803, 2006.

4. Zheng T, Holford TR, Chen Y, Ma JZ, Flannery J, Liu W, Russi M and Boyle P: Time trend and age-period-cohort effect on incidence of thyroid cancer in Connecticut, 1935-1992. Int J Cancer 67: 504-509, 1996.

5. Liu S, Semenciw R, Ugnat AM and Mao Y: Increasing thyroid cancer incidence in Canada, 1970-1996: Time trends and age-period-cohort effects. Br J Cancer 85: 1335-1339, 2001

6. Xing M: Molecular pathogenesis and mechanisms of thyroid cancer. Nat Rev Cancer 13: 184-199, 2013.

7. Begum S, Rosenbaum E, Henrique R, Cohen Y, Sidransky D and Westra WH: BRAF mutations in anaplastic thyroid carcinoma: Implications for tumor origin, diagnosis and treatment. Mod Pathol 17: 1359-1363, 2004

8. Ji S, Ye G, Zhang J, Wang L, Wang T, Wang Z, Zhang T, Wang G, Guo Z, Luo Y, et al: miR-574-5p negatively regulates Qki6/7 to impact beta-catenin/Wnt signalling and the development of colorectal cancer. Gut 62: 716-726, 2013.

9. Kondo T, Furuta T, Mitsunaga K, Ebersole TA, Shichiri M, Wu J, Artzt K, Yamamura K and Abe K: Genomic organization and expression analysis of the mouse qkI locus. Mamm Genome 10 662-669, 1999.

10. Pilotte J, Larocque D and Richard S: Nuclear translocation controlled by alternatively spliced isoforms inactivates the QUAKING apoptotic inducer. Genes Dev 15: 845-858, 2001.

11. Biedermann B, Hotz HR and Ciosk R: The Quaking family of RNA-binding proteins: Coordinators of the cell cycle and differentiation. Cell Cycle 9: 1929-1933, 2010.

12. Larocque D, Galarneau A, Liu HN, Scott M, Almazan G and Richard S: Protection of p27(Kip1) mRNA by quaking RNA binding proteins promotes oligodendrocyte differentiation. Nat Neurosci 8: 27-33, 2005.

13. Chénard CA and Richard S: New implications for the QUAKING RNA binding protein in human disease. J Neurosci Res 86 233-242, 2008

14. Yang G, Fu H, Zhang J, Lu X, Yu F, Jin L, Bai L, Huang B, Shen L, Feng Y, et al: RNA-binding protein quaking, a critical regulator of colon epithelial differentiation and a suppressor of colon cancer. Gastroenterology 138: 231-240.e1-5, 2010.

15. Ambros V: The functions of animal microRNAs. Nature 431: 350-355, 2004.
16. Bartel DP: MicroRNAs: Target recognition and regulatory functions. Cell 136: 215-233, 2009.

17. Bartel DP: MicroRNAs: Genomics, biogenesis, mechanism, and function. Cell 116: 281-297, 2004.

18. Fan M, Li X, Jiang W, Huang Y, Li J and Wang Z: A long non-coding RNA, PTCSC3, as a tumor suppressor and a target of miRNAs in thyroid cancer cells. Exp Ther Med 5: 1143-1146, 2013.

19. Melo SA and Esteller M: Dysregulation of microRNAs in cancer: Playing with fire. FEBS Lett 585: 2087-2099, 2011.

20. Barker $\mathrm{N}$ and Clevers H: Mining the Wnt pathway for cancer therapeutics. Nat Rev Drug Discov 5: 997-1014, 2006.

21. Futaki S, Suzuki T, Ohashi W, Yagami T, Tanaka S, Ueda K and Sugiura Y: Arginine-rich peptides. An abundant source of membrane-permeable peptides having potential as carriers for intracellular protein delivery. J Biol Chem 276: 5836-5840, 2001.

22. Livak KJ and Schmittgen TD: Analysis of relative gene expression data using real-time quantitative PCR and the 2(-Delta Delta C(T)) method. Methods 25: 402-408, 2001.

23. Raymond CK, Roberts BS, Garrett-Engele P, Lim LP and Johnson JM: Simple, quantitative primer-extension PCR assay for direct monitoring of microRNAs and short-interfering RNAs. RNA 11: 1737-1744, 2005.

24. Galarneau A and Richard S: Target RNA motif and target mRNAs of the Quaking STAR protein. Nat Struct Mol Biol 12: 691-698, 2005.

25. Shenouda SK and Alahari SK: MicroRNA function in cancer: Oncogene or a tumor suppressor? Cancer Metastasis Rev 28: 369-378, 2009.

26. Li Q,Li X, Guo Z, Xu F, Xia J, Liu Zand Ren T: MicroRNA-574-5p was pivotal for TLR9 signaling enhanced tumor progression via down-regulating checkpoint suppressor 1 in human lung cancer. PLoS One 7: e48278, 2012.

27. Noveroske JK, Lai L, Gaussin V, Northrop JL, Nakamura H, Hirschi KK and Justice MJ: Quaking is essential for blood vessel development. Genesis 32: 218-230, 2002.

28. Novikov L, Park JW, Chen H, Klerman H, Jalloh AS and Gamble MJ: QKI-mediated alternative splicing of the histone variant MacroH2A1 regulates cancer cell proliferation. Mol Cell Biol 31: 4244-4255, 2011.

29. Bian Y, Wang L, Lu H, Yang G, Zhang Z, Fu H, Lu X, Wei M, Sun J, Zhao Q, et al: Downregulation of tumor suppressor QKI in gastric cancer and its implication in cancer prognosis. Biochem Biophys Res Commun 422: 187-193, 2012.

30. Zong FY, Fu X, Wei WJ, Luo YG, Heiner M, Cao LJ, Fang Z, Fang R, Lu D, Ji H and Hui J: The RNA-binding protein QKI suppresses cancer-associated aberrant splicing. PLoS Genet 10: e1004289, 2014.

31. He Z, Yi J, Liu X, Chen J, Han S, Jin L, Chen L and Song H: MiR-143-3p functions as a tumor suppressor by regulating cell proliferation, invasion and epithelial-mesenchymal transition by targeting QKI-5 in esophageal squamous cell carcinoma. Mol Cancer 15: 51, 2016.

32. Miyoshi K, Rosner A, Nozawa M, Byrd C, Morgan F, Landesman-Bollag E, Xu X, Seldin DC, Schmidt EV, Taketo MM, et al: Activation of different Wnt/beta-catenin signaling components in mammary epithelium induces transdifferentiation and the formation of pilar tumors. Oncogene 21 : 5548-5556, 2002.

33. Wieczorek M, Paczkowska A, Guzenda P, Majorek M, Bednarek AK and Lamparska-Przybysz M: Silencing of Wnt-1 by siRNA induces apoptosis of MCF-7 human breast cancer cells. Cancer Biol Ther 7: 268-274, 2008. 